\title{
Schrift als Komponente der Bildschirmfläche am Beispiel der deutschen Nachrichtensendungen
}

\author{
Anna Kapuścińska (Bydgoszcz)
}

\begin{abstract}
The article focusses the written components of the screen surface in television news programs. The semiotic perspective represented in this article legitimises the viewpoint that they are not (ordinary) language signs. The main concern of this article is the status of the written text (as a semiotic medium) in the construction patterns of the news programs. It is argued that the written texts appear in the news programs increasingly as graphical elements, so that their visual presence becomes primary to their language meaning. This tendency is discussed in the article on the example of three news programs in the German television between 1996 and 2016.
\end{abstract}

\section{$1 \quad$ Einleitung}

Das Hauptanliegen des vorliegenden Beitrags ist die vergleichende Analyse des evolvierenden Status von dem (semiotischen) Medium - (Schrift)Sprache in dem (technischen) Medium Fernsehen, und zwar in dem (kulturbezogenen) Medium - Nachrichtensendungen zu betrachten. Obwohl die Konstruktion der Bildschirmfläche, also auch die Gestaltung und Platzierung der Schriftelemente relativ stabilen Schemata unterliegen, lassen sich in der mikro-diachronen Perspektive sichtbare Veränderungen und folgerichtig Entwicklungstendenzen im Gebrauch der Schrift in den Nachrichtensendungen anzeigen. Diese werden in dem vorliegenden Beitrag anhand von den Vorkommensweisen der Schrift auf der Bildschirmfläche in ausgewählten deutschen Nachrichtensendungen aus den Jahren 1996 und 2011 und 2016 diskutiert.

\section{$2 \quad$ Verschiedene Begriffe des Mediums}

Zur terminologischen Klärung dieser Zielsetzung erscheint es als fruchtbar, den hierin dreifach gebrauchten Begriff Medium näher zu betrachten. Eine Basis dafür bildet das Konzept des Medienbegriffs von Roland Posner. Unter fünf unterschiedlichen Begriffen von Medium nach Posner werden $u$. a. der technische, semiotische und kulturbezogene Medienbegriff genannt.

Der erstere „charakterisiert die Zeichensysteme nach den technischen Medien, die bei der Erzeugung von Zeichenprozessen zur Modifikation der Kontaktmaterie eingesetzt werden, die die physische Verbindung zwischen dem Produktionsorgan des Senders und dem Rezeptionsorgan des Empfängers herstellt" (Posner 1985: 256). Zu den visuellen Medien im technologischen Sinn gehören nach Posner u. a. Bleistift, Papier, Brille, Opernglas oder Fernrohr, sowie auch solche Geräte, die zur Produktion oder Wiedergabe kommunikativer Artefakte dienen können, darunter Schreibmaschinen, Fotokameras, Videokameras, wie auch 
Overheadprojektoren oder Mikrofilme. Darunter unterscheidet Posner je nach der Art des Produkts Printmedien, Projektionsmedien und Bildschirmmedien (cf. ibd.). Zu der letzten Kategorie ist auch das Fernsehen zu zählen, das in dem vorliegenden Beitrag betrachtet wird.

Der semiotische, kodebezogene Medienbegriff betrifft hingegen „die Zeichensysteme nach den Regeln, mit Hilfe derer ihre Benutzer bei der Zeichenproduktion den Botschaften Zeichenträger und bei der Rezeption den Zeichenträgern Botschaften zuordnen“ (ibd.: 257). Die Entscheidungen zwischen verschiedenen Medien sind demnach „,[d]ie Entscheidung eines Verlags, ob er ein Buch auf Deutsch, Englisch oder Französisch herausgibt, eines Komponisten, ob er tonal, oder atonal komponiert, eines Malers, ob er gegenständlich oder nichtgegenständlich malt, oder eines Architekten, ob er ein Haus im neuromantischen, neugotischen oder neusachlichen Stil baut"، (ibd.).

Schließlich charakterisiert der kulturbezogene Medienbegriff „die Zeichensysteme nach dem Zweck der Botschaften, die durch sie vermittelt werden“" (ibd.: 268). Als Beispiele für Medien nach dem kulturbezogenen Begriff nennt Stöckl „Werbung, Glosse, Lyrik oder Romane“ (Stöckl 2010: 3), wobei „der Verfahrens- oder kulturelle Routinen-Charakter des Medialen“ (ibd.) in den Vordergrund tritt. Diese Definition lässt den kulturbezogenen Medienbegriff mit dem linguistischen Begriff der Kommunikationsform (cf. Dürscheid 2011: 94f.) identifizieren.

Eine kritische Betrachtung der unterschiedlichen Medienbegriffe von Posner u. a. bei Schmitz macht deutlich, dass, obwohl selbst die Sprache als ein Medium gelten kann (cf. Jäger 2001; cf. auch Schmitz 2015: 8), in der (medien)linguistischen Forschung ${ }^{1}$ der technische ${ }^{2}$ Medienbegriff dominiert, wonach ein Medium ein ,zur Kommunikation verwendetes Gerät, z. B. ein Smartphone“ (Schmitz 2015: 8) ist.

Die Dominanz des technischen Medienbegriffs in der Sprachwissenschaft wird auf die strukturalistische Tradition in der Auffassung der Sprache zurückgeführt. Nach Püschel ist eine „ausdrückliche Herausstellung des Mediencharakters von Sprache und ihre Einbeziehung in die linguistische Theoriebildung [...] für die Linguistik [...] nicht als selbstverständlich und von vornherein gegeben anzusehen“" (Püschel 1975: 3). Von de Saussure wird sie überhaupt nicht hervorgehoben, obwohl er langue als ein grammatisches System definiert, „über das die Sprecher verfügen“ (cf. de Saussure 1967: 16; cf. auch Püschel 1975: 3).

In den gegenwärtigen linguistisch basierten Ansätzen, die ebenso den technischen Medienbegriff annehmen, hat sich vielmehr die Ansicht etabliert, „dass das Medium [nicht] nur ein Hilfsmittel ist, das dazu dient, Distanzen in der Kommunikation zu überwinden und die Botschaft vom Sender zum Empfänger zu transportieren“ (Dürscheid 2011: 94), sondern auch die Botschaft mitgestalten kann. Dennoch hat die grundsätzliche Einigung der meisten Linguisten, die sich mit der Thematik der Medien befassen, über den technischen Medienbegriff beträchtliche Folgen für die linguistisch basierte Mediendiskussion. Ein Vorteil davon sei nach Schneider (2006: 75), dass dadurch terminologische Eindeutigkeit gewährleistet wird. Ein Nachteil

\footnotetext{
${ }^{1}$ Zur Medienlinguistik als einer linguistischen Forschungsrichtung cf. Kapuścińska (2016).

2 In Bezug auf den Medienbegriff von Posner verwendet man in der einschlägigen Literatur auch die Bezeichnung „technologisch“ (so z. B. Stöckl 2010: 2). In diesem Beitrag wird jedoch nach Posner der Begriff „technisch“ verwendet. Die beiden Begriffe sind als Synonyme zu betrachten. Zur Diskussion des Begriffs technischer Medien cf. auch Winkler 2008: 91-131(cf. auch Schneider 2017: 36-39).
} 
sei hingegen, dass die terminologische Eindeutigkeit ,zu teuer erkauft“ ist, indem man Gefahr läuft, die Rolle der Sprache zu einer Größe zu reduzieren, die „,in Medien oder durch Medien transportiert wird“ (ibd., Hervorhebung im Original; cf. auch Schneider 2017: 35f.). ${ }^{3}$

Diese Gefahr, die im vergleichbaren Ausmaß für die Sprache (als das semiotische Medium) und für die Kommunikationsformen (als das kulturbezogene Medium) gilt, erscheint umso größer, dass der in der Linguistik etablierte technische Medienbegriff im Vergleich zum technischen Medienbegriff nach Posner verengt ist. Erstens wird der erstere (auch wenn nur implizit) auf die elektronischen Medien beschränkt, wodurch „Medien“ wie Bleistift, Papier oder Brille außer Acht gelassen werden. Zweitens werden in den meisten medienlinguistischen Bearbeitungen nur die sog. „Massenmedien“ und nicht „,interpersonale Medien“ fokussiert (cf. Schmitz 2015: 12). Während sich die Grenze zwischen den (elektronischen) Massenmedien und interpersonalen Medien nur schwer ziehen lässt (cf. Dürscheid 2007), wäre vielmehr davon auszugehen, dass die linguistische Perspektive nur ausgewählte Anwendungen der Medien umfasst, und zwar diejenigen, die sie als die Massenmedien konstituieren.

Die Dissonanz zwischen dem medienlinguistischen technischen Medienbegriff und dem breiteren technischen Medienbegriff nach Posner wirkt sich dennoch kaum auf die vorliegende Diskussion um das (technische) Medium Fernsehen aus. Selbst bei der Annahme des engeren Begriffs vom technischen Medium wird das Fernsehen problemlos davon erfasst, weil es zweifelsohne ein elektronisches Massenmedium ist. Es funktioniert nämlich nur unter Stromversorgung und die damit vermittelte Kommunikation ist in der Regel an mehrere Empfänger gerichtet.

Zugleich werden hier jedoch auch die (Schrift)Sprache und die Nachrichtensendungen als (entsprechend) semiotische und kulturbezogene Medien betrachtet. Dadurch soll die oben erwähnte Illusion vermieden werden, als wären die Schrift und Nachrichtensendungen nur transportierte Größen. Vielmehr werden sie zu Medien aufgewertet, die sich beide auf die Gestaltung der Botschaft auswirken.

\section{Schrift als semiotisches Medium in den Nachrichtensendungen}

Die Zielsetzung dieses Beitrags erzwingt zweierlei Einschränkungen der Sichtweite. Erstens wird hier nur das semiotische Medium analysiert, das sich ohnehin im Spannungsfeld der technischen und kulturbezogenen Medialität befindet. Zweitens wird das Augenmerk grundsätzlich nur auf ein semiotisches Medium gerichtet. Die zweite Einschränkung rückt die Vorwarnung von Werner Holly in den Vordergrund, dass ein Sprachwissenschaftler die Sprache nicht aus dem ganzen kommunikativen Kontext herausnehmen und isoliert betrachten soll (cf. Holly 2004: 159). Diese durchaus annehmbare Behauptung wird hierin keinesfalls bestritten. Es wird hier auch auf keinen Fall versucht, nur das (Schrift)Sprachliche abzustecken, ohne gleichzeitige Betrachtung der nichtsprachlichen Komponenten des multimedialen (audiovisuellen) Kommunikats.

\footnotetext{
3 Diese übliche Auffassung von Medium in der Linguistik entspricht nach Schneider (2006: 78) nicht der Etymologie des Wortes Medium, nach dem das Medium nicht nur ein Ding sondern auch ein Verfahren ist. „Etymologisch betrachtet, kann das lateinische Wort ,Medium‘ das Zitat ist richtig sowohl (räumliche) ,Mitte', als auch ,Mittler` bzw. ,Vermittelndes‘ (funktional), als auch ,Mittel‘ bzw. ,Werkzeug‘ (final) bedeuten“ (ibd.).
} 
Die Tatsache, dass die vorliegende Analyse keine nichtsprachlichen Einheiten umfasst, die von einer Nachrichtensendung unabdingbar sind, ist ausschließlich durch den Vorsatz bedingt, sich in dem Beitrag auf das semiotische Medium Schrift mit seinen ausgewählten Vorkommensweisen und Funktionen in den kulturbezogenen Medium Kommunikationsform Nachrichtensendung zu konzentrieren.

Anzumerken ist, dass die Schrift eine besonders untersuchungswerte Komponente der Nachrichtensendung vor allem aus der Perspektive der Sprachwissenschaft darstellt. Sie scheint nämlich semiotisch gesehen ihren primären Signifizierungswert zumindest partiell eingebüßt zu haben. Auch in den sprachwissenschaftlichen Arbeiten sind die Ansichten anzutreffen, dass man es in solchen Kommunikationsformen mit einer nicht traditionellen Signifikation zu tun habe. Dieses Phänomen wird von Schmitz als die ,tertiäre Schriftlichkeit“ bezeichnet und gilt damit als „eine dritte Art von Schriftlichkeit“ (Schmitz 2015: 46) im Vergleich zu der primären und sekundären Schriftlichkeit. Während unter der primären Schriftlichkeit die „klassische“ Schriftlichkeit gemeint wird, definiert Schmitz die sekundäre Schriftlichkeit als die halbspontane Art der Schriftlichkeit, die sich im Zeitalter der digitalen Medien durch „schriftliche Dia- und Multiloge“ (ibd.: 45) manifestiert. „Auf der Grundlage schriftgewohnter Reflektiertheit wird gewollte Spontaneität zugelassen, oder gar kultiviert“ (ibd.). Schmitz zufolge gab es solche Schriftlichkeit ,früher nur in exotischen Ausnahmefällen, etwa bei heimlichen SchülerKassibern (Zettelpost) im Schulunterricht"، (ibd.). Einfacher gefasst, wird damit die mediale Schriftlichkeit bei gleichzeitiger konzeptioneller Mündlichkeit gemeint (cf. Koch/Oesterreicher 2011: 3). Wie zurecht festgestellt wird, ist diese Art der Schriftlichkeit heutzutage wesentlich weiter verbreitet, wie z. B. in der Chat- oder SMS-Kommunikation.

Die tertiäre Schriftlichkeit ist hingegen eine Schriftlichkeit, bei der „nicht auf den Wortlaut allein ankommt, sondern [...] die visuelle Erscheinung und Umgebung von Schrift eine Rolle für ihr Verständnis spielt" (Schmitz 2015: 46). Sie ergeben sich grundsätzlich aus der Bemühung, ,auf kleiner Fläche möglichst viel Informationen so darzustellen, dass sie (am besten selektiv) möglichst schnell erfasst werden kann“ (ibd.: 115). Solche visuellen Botschaften treten in Form von „Sehflächen“" auf, die bereits zehn Jahre früher von Schmitz thematisiert worden sind (cf. Schmitz 2005). Durch die komplexen Beziehungen zwischen solchen Elementen wie „Layout, Bilder, Texte, Wörter und andere sichtbare Zeichen“ (Schmitz 2010: 400) nimmt die Schrift „einige Eigenschaften von Bildern an“ (ibd.).

Der grundlegende Unterschied zwischen den drei Arten der Schriftlichkeit wird wie folgt erläutert:

Während in primärer Schriftlichkeit (z. B. in einem Roman) alle gemeine Bedeutung situationsunabhängig schriftlich formuliert werden muss und während in sekundärer Schriftlichkeit (ähnlich wie in primärer Oralität) Bedeutung nach und nach dialogisch entwickelt wird, wird Bedeutung in tertiärer Schriftlichkeit auf den Wortlaut, seine Gestaltung (Typographie) und Platzierung sowie sein Zusammenspiel mit dem zugehörigen Bildanteil verteilt.

(Schmitz 2015: 46)

$\mathrm{Ob}$ die primäre Schriftlichkeit tatsächlich ,situationsunabhängig“ ist, erscheint dabei als diskutabel. U. a. von Fix (2008) wird auf die wesentliche Rolle der Lokalität geschriebener Sprache hingewiesen. 
Als Lokalität wird der Ort der Publikation gemeint, in Abgrenzung von dem Ort der Produktion und Rezeption sowie von der „Darstellung von Raumverhältnissen im Text oder mit dem Raumbezug der Interaktionspartner“ (ibd.: 349). Nach Fix ist der Ort auch nicht dem Material als Textträger gleichzusetzen, wie „Papier, Stein, Textilien“ (ibd.). Dazu gehört auch das „,institutionalisierte“ Material: „Papier als Buch, als Zeitschrift oder als Flugblatt, Stein als Gedenkstein, Steine als Mauer eines bestimmten Gebäudes, Textilien als T-Shirt oder Mütze“ (ibd.). Nach Fix kann der Ort drei verschiedene Funktionen für einen Text bzw. eine Textsorte ausüben: „Hervorhebung und Intensivierung einer Textfunktion“, „Bedingung für eine Textsorte an sich“ und „Bedingung für die Textsortenzuordnung im konkreten Fall“ (ibd.: 349f.). Jedenfalls ist es jedoch der Differenzierung von Schmitz zu entnehmen, dass die sprachliche Kodierung bei der tertiären Schriftlichkeit einen geringeren Teil des Kommunikats ausmacht als bei den zwei sonstigen „Schriftlichkeiten“, weil das Kommunikat zugleich durch das Sprachliche und (nicht weniger) das Nichtsprachliche vermittelt wird.

In das Konzept der tertiären Schriftlichkeit fügt sich auch die Beobachtung, dass die Schrift (als eine Komponente der Sehfläche) in gewissen kommunikativen Umgebungen ihre sprachlich kodierte Signifikation völlig zu verlieren scheint. Cieszkowski zufolge wird, aus der Sprache das Symbolische entfernt“, indem sie als eine Komponente eines präzisen, technisch bearbeiteten Schemas ,zu einem Ding reduziert [wird], das mit anderen Dingen vergleichbar ist“" (cf. Cieszkowski 2010: 183; cf. auch Kapuścińska 2017: 127f.). ${ }^{4}$

\section{Schriftgebrauch in deutschen Nachrichtensendungen im Zeitwandel}

Vor dem Hintergrund der theoretischen Ausführungen wird im Folgenden die Anwesenheit der Schrift in drei deutschen Nachrichtensendungen: tagesschau, ZDF heute und RTL aktuell im Zeitraum von zwanzig Jahren diskutiert. Dabei werden drei Zeitpunkte in Betracht gezogen. Der erstere ist das Jahr 1996. Zwei weitere Punkte sind nur fünf Jahre voneinander entfernt. Dies hat zum Ziel, zu überprüfen, ob auch in einem so relativ kurzen Zeitraum Unterschiede im Schriftgebrauch sichtbar sind. ${ }^{5}$ Es wird davon ausgegangen, dass die Schriften auf der Bildschirmfläche, die den Nachrichtensendungen erst in der Postproduktion als Einblendungen hinzugefügt worden sind, ${ }^{6}$ prinzipiell die tertiäre Schriftlichkeit aufweisen. Ihr Status auf der Skala der Relevanz von dem Symbolischen (Sprachlichen) bis hin zu einem nahezu völligen Relevanzverlust ist mit dem Grad der Schematizität in der Gestaltung des Bildschirms gekoppelt. Der (eventuelle) Wandel von diesem Status wird hier anhand von vier Schriftelementen verfolgt: Hyperlink, Nachrichtenticker, Logotype und Personenbezeichnungsfläche.

\footnotetext{
${ }^{4}$ Solche schriftbasierten Einheiten können als „Text/Bilder“ (cf. Cieszkowski 2014: 41) oder (deskriptiv) als Grenzphänomene zwischen Text und Bild bezeichnet werden (cf. Kapuścińska 2017).

${ }^{5}$ Eine ausführlichere Analyse der Gestaltungsschemata in den deutschen Nachrichtensendungen im Jahr 2011 ist Kapuścińska (2017) zu entnehmen.

${ }^{6}$ Das unterscheidet sie von den Einheiten, die zum Videomaterial gehören, bei denen die primäre und sekundäre Schriftlichkeit dominieren.
} 


\begin{tabular}{|l|c|l|l|}
\hline \multirow{2}{*}{} & \multicolumn{3}{|c|}{ Veränderungen im Zeitraum von 1996 bis 2016 } \\
\cline { 2 - 4 } & 1996 & \multicolumn{1}{|c|}{2011} & \multicolumn{1}{|c|}{2016} \\
\hline $\begin{array}{l}\text { Das Erste } \\
\text { tages- } \\
\text { schau) }\end{array}$ & - & $\begin{array}{l}\text { transparenter Streifen im unteren Bereich mit dem Sender- } \\
\text { namen sowie dem Text ,Mehr Informationen unter } \\
\text { www.tages-schau.de und im Infokanal tagesschau24“ in } \\
\text { weißer Schrift (cf. Kapuścińska 2017: 214) }\end{array}$ & $\begin{array}{l}\text { keine } \\
\text { Verände- } \\
\text { rung zu } \\
2011\end{array}$ \\
\hline $\begin{array}{l}\text { RTL } \\
\text { RTL } \\
\text { aktuell) }\end{array}$ & - & $\begin{array}{l}\text { kurzer grauer Streifen unten links mit einem rot-orangen } \\
\text { oberen Rand und dem Text „mehr Infos bei: } \\
\text { www.RTL.de“ in dunkelgrauer und (Adresse) roter Schrift } \\
\text { (cf. Kapuścińska 2017: 213) }\end{array}$ & $\begin{array}{l}\text { keine } \\
\text { Verände- } \\
\text { rung zu } \\
\text { 2011 }\end{array}$ \\
\hline $\begin{array}{l}\text { ZDF } \\
\text { ZDF } \\
\text { heute) }\end{array}$ & - & $\begin{array}{l}\text { grau-oranges Streifen im unteren Bereich mit der weißen } \\
\text { Schrift: der Sendungslogotype sowie der Internet-Adresse } \\
\text { und Teletext-Seite (cf. Kapuścińska 2017: 212) }\end{array}$ & $\begin{array}{l}\text { keine } \\
\text { Verände- } \\
\text { rung zu } \\
\text { 2011 }\end{array}$ \\
\hline
\end{tabular}

Tabelle 1: Nachrichtensendungen: Hyperlink

Die Gestaltungsweise der Hyperlinks in den analysierten Beispielen zeigt sich als relativ stabil und weist keine Veränderungen innerhalb von den letzten fünf Jahren auf. Die Tatsache, dass es im Jahr 1996 keine Hyperlinks gab, ist wenig überraschend und ergibt sich aus dem damaligen Stand der technischen Entwicklung. Eine allgemeine Tendenz, die sich in allen drei Sendungen beobachten lässt, ist eine relativ geringe Auffälligkeit der Hyperlinks. Sie erscheinen jeweils auf streifenartigen Flächen, deren Farbe wenig kontrastiv in Bezug auf die Farben im Fernsehstudio sind. Auch die Schriftfarben (ausgenommen die rote Schrift bei RTL) sind eher abgetönt. Explizit ist ebenfalls eine vorherrschende Tendenz in der Platzierung der Hyperlinks in den Nachrichtensendungen, und zwar sowohl in räumlicher als auch in zeitlicher Hinsicht. Räumlich gesehen befindet sich der Hyperlink (als ein Element einer satzwertigen oder nicht satzwertigen Äußerung) im unteren Bereich der Bildschirmfläche, der ein üblicher Ort auch für andere streifenartige Einblendungen ist. In der zeitlichen Hinsicht wird der Hyperlink prinzipiell am Ende der Sendung, entweder während der mündlichen Verabschiedung des Moderators oder direkt danach angezeigt.

\begin{tabular}{|c|c|c|c|}
\hline & \multicolumn{3}{|c|}{ Veränderungen im Zeitraum von 1996 bis 2016} \\
\hline & 1996 & 2011 & 2016 \\
\hline $\begin{array}{l}\text { Das } \\
\text { Erste } \\
\text { (tages- } \\
\text { schau) }\end{array}$ & - & - & - \\
\hline $\begin{array}{l}\text { RTL } \\
\text { (RTL } \\
\text { aktuell) }\end{array}$ & - & $\begin{array}{l}\text { blauer Streifen (während der Werbung direkt vor dem Anfang der } \\
\text { Sendung) mit der Logotype der Sendung, dem Sekundenzähler } \\
\text { und News-Ticker in weißer Schrift (cf. Kapuścińska 2017: 246) }\end{array}$ & - \\
\hline $\begin{array}{l}\text { ZDF } \\
\text { (ZDF } \\
\text { heute) }\end{array}$ & - & - & - \\
\hline
\end{tabular}

Tabelle 2: Nachrichtenticker 
Der Nachrichtenticker, der sich nach der „Revolution“ bei CNN im Sommer 2001 als eine Komponente der Nachrichtensendungen etabliert hat (cf. Kapuścińska 2016: 26), kommt vorwiegend in den Spartenprogrammen mit Schwerpunkt Nachrichten vor. Seltener ist er in den Vollprogrammen (wie die hier betrachteten) anwesend, was mit der obigen Zusammenstellung bestätigt wird. Selbst in dem einzigen Beispiel eines Nachrichtentickers ist von seiner eher marginalen Bedeutung auszugehen. Erstens wird er nicht während der eigentlichen Laufzeit der Sendung angezeigt, sondern davor, während der Präsentation einer Werbung, und zweitens wird 2016 bereits auf diese Komponente verzichtet, obwohl die sonstige Gestaltung der Sendung kaum Veränderungen aufweist. Dieses Beispiel bestätigt jedoch gleichzeitig die frühere Behauptung, dass die Streifen mit jeglichen Schrifteinheiten in der Regel im unteren Bereich des Bildschirms platziert sind.

\begin{tabular}{|c|c|c|c|}
\hline & \multicolumn{3}{|c|}{ Veränderungen im Zeitraum von 1996 bis 2016} \\
\hline & 1996 & 2011 & 2016 \\
\hline $\begin{array}{l}\text { Das } \\
\text { Erste } \\
\text { (tages- } \\
\text { schau) }\end{array}$ & $\begin{array}{l}\text { Senderlogotype nur in } \\
\text { der Vorspannanima- } \\
\text { tion; farbige, gelbe } \\
\text { Sendungslogotype in } \\
\text { der Vorspannanima- } \\
\text { tion und danach weiße } \\
\text { Sendungslogotype } \\
\text { oben in der Hinter- } \\
\text { grundgraphik }\end{array}$ & $\begin{array}{l}\text { monochrome Senderlogotype } \\
\text { (leicht verändert im Vergleich zu } \\
\text { 1996) oben rechts; weiße Sen- } \\
\text { dungslogotype (leicht verändert im } \\
\text { Vergleich zu 1996) in der Vorspan- } \\
\text { nanimation, in der Hintergrundgra- } \\
\text { phik sowie in allen streifenartigen, } \\
\text { die während der Sendung gezeigt } \\
\text { werden (cf. Kapuścińska 2017: } \\
\text { 196) }\end{array}$ & $\begin{array}{l}\text { stets angezeigte } \\
\text { Senderlogotype } \\
\text { unten links; weiße } \\
\text { Sendungslogotype } \\
\text { in der Vorspanna- } \\
\text { nimation, keine } \\
\text { Logotype in der } \\
\text { Hintergrundgra- } \\
\text { phik }\end{array}$ \\
\hline $\begin{array}{l}\text { RTL } \\
\text { (RTL } \\
\text { aktuell) }\end{array}$ & $\begin{array}{l}\text { monochrome Sender- } \\
\text { logotype oben links; } \\
\text { farbige Sendungslo- } \\
\text { gotype in der Vor- } \\
\text { spannanimation }\end{array}$ & $\begin{array}{l}\text { monochrome Senderlogotype oben } \\
\text { links; farbige Sendungslogotype } \\
\text { (stark verändert im Vergleich zu } \\
\text { 1996) in dem blauen Streifen des } \\
\text { Nachrichtentickers vor der Sen- } \\
\text { dung sowie in der Vorspannanima- } \\
\text { tion und in der Hintergrundgraphik } \\
\text { am Angang der Sendung }\end{array}$ & $\begin{array}{l}\text { keine Verände- } \\
\text { rung zu } 2011\end{array}$ \\
\hline $\begin{array}{l}\text { ZDF } \\
\text { (ZDF } \\
\text { heute) }\end{array}$ & $\begin{array}{l}\text { farbige (blaue) Sen- } \\
\text { derlogotype unten in } \\
\text { der Hintergrundgra- } \\
\text { phik; farbige, grau- } \\
\text { blaue Sendungslogo- } \\
\text { type in der Vorspan- } \\
\text { nanimation und da- } \\
\text { nach oben in der Hin- } \\
\text { tergrundgraphik }\end{array}$ & $\begin{array}{l}\text { monochrome Senderlogotype oben } \\
\text { links (stark verändert im Vergleich } \\
\text { zu 1996); farbige, orange-weiße } \\
\text { Sendungslogotype (ebenso stark } \\
\text { verändert im Vergleich zu 1996) in } \\
\text { der Animation in der Hintergrund- } \\
\text { graphik in der Anfangsphase (cf. } \\
\text { Kapuścińska 2017: 169) }\end{array}$ & $\begin{array}{l}\text { keine Verände- } \\
\text { rung zu } 2011\end{array}$ \\
\hline
\end{tabular}

Tabelle 3: Logotype

Unter den Logotypen werden oben zwei Gruppen differenziert. Die erste sind die Senderlogotypen. Sowohl 2011 als auch 2016 war es üblich, dass die Senderlogotype, zumeist in einer monochromen Version durch die ganze Laufzeit der Sendung (oder sogar über den Rahmen der Sendung hinaus) im oberen Bereich der Bildschirmfläche, entweder links oder rechts angezeigt 
wurde. Eine Ausnahme bildet nur ZDF heute im Jahr 2016, dessen Produzenten darauf verzichteten. Diese Tendenz ist 1996 noch nicht so verbreitet. Eine solche Anwendung der Senderlogotype lässt in nur einer von drei Nachrichtensendungen von 1996 beobachten, die hierbei der Analyse unterzogen sind, i. e. in RTL aktuell. Dabei können die Senderlogotypen im Laufe der Zeit bestimmten Veränderung unterliegen. Von den analysierten Beispielen kann eine besonders markante Veränderung zwischen dem Jahr 1996 und 2011 bei ZDF beobachtet werden. Ganz unverändert ist hingegen in diesem Zeitraum die Logotype von RTL geblieben. Veränderungen der Logotype zwischen 2011 und 2016 gibt es bei keinem Sender, was von einer relativen Stabilität dieses Elements zeugen kann.

Eine ähnliche Beobachtung lässt sich in Bezug auf die zweite Gruppe: die Sendungslogotypen machen. Keine davon ist zwischen 1996 und 2011 ohne Veränderung geblieben, wobei in zwei Fällen die Veränderung besonders weitgehend ist. Ein gemeinsames Merkmal aller analysierten Sendung ist, dass die Sendungslogotype immer als ein Element der Vorspannanimation verwendet wird. Erst in den Sendungen von 2011 erscheint sie hingegen als eine obligatorische Komponente der Hintergrundgraphik (mindestens in der Anfangsphase der Sendung).

\begin{tabular}{|c|c|c|c|}
\hline & \multicolumn{3}{|c|}{ Veränderungen im Zeitraum von 1996 bis 2016} \\
\hline & 1996 & 2011 & 2016 \\
\hline $\begin{array}{l}\text { Das } \\
\text { Erste } \\
\text { (tages- } \\
\text { schau) }\end{array}$ & $\begin{array}{l}\text { Personenbezeichnungs- } \\
\text { fläche als ein blauer } \\
\text { Streifen unten mit wei- } \\
\text { ßer Schrift; die Perso- } \\
\text { nenbezeichnung des } \\
\text { Moderators nur als eine } \\
\text { weiße Schrift unten } \\
\text { rechts (mit dem Zusatz } \\
\text { „Sprecher“ bzw. „Spre- } \\
\text { cherin“) }\end{array}$ & $\begin{array}{l}\text { Personenbezeich-nungsflä- } \\
\text { che als ein blauer Streifen } \\
\text { unten mit der animierten } \\
\text { Sendungslogotype und sowie } \\
\text { mit weißer Schrift; die Per- } \\
\text { sonenbezeichnung des Mo- } \\
\text { derators nur als eine weiße } \\
\text { Schrift unten links (cf. Ka- } \\
\text { puścińska 2017: 287f.) }\end{array}$ & $\begin{array}{l}\text { Personenbezeich- } \\
\text { nungsfläche als ein } \\
\text { dunkelblauer Streifen } \\
\text { unten mit weißer } \\
\text { Schrift (die Sendungs- } \\
\text { logotype stets ange- } \\
\text { zeigt); die gleiche Ge- } \\
\text { staltung auch bei der } \\
\text { Personenbezeichnung } \\
\text { des Moderators }\end{array}$ \\
\hline $\begin{array}{l}\text { RTL } \\
\text { (RTL } \\
\text { aktuell) }\end{array}$ & $\begin{array}{l}\text { keine Bezeichnungen } \\
\text { der gerade sprechenden } \\
\text { Personen; Personenbe- } \\
\text { zeichnungsfläche des } \\
\text { Moderators als ein } \\
\text { blaues Streifen unten } \\
\text { mit der animierten Sen- } \\
\text { dungslogotype und so- } \\
\text { wie mit weißer Schrift }\end{array}$ & $\begin{array}{l}\text { Personenbezeichnungsfläche } \\
\text { als ein blaues Streifen unten } \\
\text { mit der animierten Sen- } \\
\text { dungslogotype und sowie } \\
\text { mit weißer Schrift (ganz ver- } \\
\text { ändert im Vergleich zu 1996; } \\
\text { cf. Kapuścińska 2017: 297) }\end{array}$ & $\begin{array}{l}\text { keine Veränderung zu } \\
2011\end{array}$ \\
\hline $\begin{array}{l}\text { ZDF } \\
\text { (ZDF } \\
\text { heute) }\end{array}$ & $\begin{array}{l}\text { Personenbezeichnungen } \\
\text { nur als eine gelbe } \\
\text { Schrift unten }\end{array}$ & $\begin{array}{l}\text { Personenbezeichnungsfläche } \\
\text { als ein grau-oranges Streifen } \\
\text { unten links mit weißer } \\
\text { Schrift - der Sendungslogo- } \\
\text { type sowie dem Vor- und } \\
\text { Nachnamen (cf. Ka- } \\
\text { puścińska 2017: 289) }\end{array}$ & $\begin{array}{l}\text { keine Veränderung zu } \\
2011\end{array}$ \\
\hline
\end{tabular}

Tabelle 4: Personenbezeichnungsfläche 
Die Analyse lässt ein konstantes Merkmal in der Platzierung der Personenbezeichnungen feststellen. Sie sind nämlich im Prinzip im unteren Bereich des Bildschirms lokalisiert. Abgesehen davon lässt sich eine Reihe von Unterschieden beobachten. Es ist anhand von den analysierten Beispielen sichtbar, dass dieses Element um 1996 nicht in allen Sendungen verwendet wurde, während es in den neueren Ausgaben als nahezu obligatorisch gilt. Früher hatte die Personenbezeichnung meistens nur die Form einer eingeblendeten Schrift, während sie sich heute (obwohl auch nicht ausnahmslos) auf einer (oft halbtransparenten) streifenartigen Fläche befindet.

\section{Schlussbemerkungen}

Die obige Analyse macht deutlich, dass sich der Status der Schriftelemente in den Nachrichtensendungen innerhalb von den zwanzig Jahren (1996-2016) deutlich verändert hat. Die größte Veränderung ist zwischen den zwei ersten Zeitpunkten (1996 und 2011) zu sehen. Es kann möglicherweise daran liegen, dass die analysierten Veränderungen sprunghaft erfolgten, und dass es zwischen den Jahren 1996 und 2011 eine Wende in der Strukturierung der Nachrichtensendungen in Deutschland gab. Dies könnte mit der besagten „Revolution“ bei CNN im Sommer 2001 verbunden sein. Die durchgeführte Analyse lässt diese Vermutung jedoch weder anlehnen noch belegen. Immerhin lässt es sich mit dem temporalen Faktor begründen, dass die Veränderungen in dem Zeitraum von nur fünf Jahren (2011 und 2016) nicht so sichtbar wie in dem Zeitraum von fünfzehn Jahren (1996 und 2011) sind.

Bei allen drei Zeitpunkten lässt sich von der tertiären Schriftlichkeit solcher Texte ausgehen, weil sie nach wie vor bestimmten Schemata unterliegen. Auch im Jahre 1996 waren die damals noch ziemlich kargen Schriftelemente auf der Fläche des Bildschirms einem Schema unterordnet, dass ihre typographische Gestaltung sowie genaue Platzierung festlegten. Es ist nämlich keinesfalls als ein Zufall zu betrachten, dass z. B. die Personenbezeichnungen in den analysierten Sendungen immer im unteren Bereich des Bildschirms angezeigt werden. Aus diesem Grund kommt dabei zweifelsohne die tertiäre Schriftlichkeit zum Einsatz. Da aber die damaligen visuellen Schemata der Nachrichtensendungen relativ wenige Komponenten enthalten, lässt sich bei den darin enthaltenen Schriftelementen eine hohe Relevanz vom Wortlaut voraussetzen. Anders ist es bei der tertiären Schriftlichkeit der Schriftelemente zu den späteren Zeitpunkten. Eine völlige Auflösung des Sprachlichen zugunsten des Visuellen lässt sich nicht arbiträr festlegen, weil sie zum Teil auch von subjektiven, empfängerbedingten Faktoren abhängt. Dennoch führt die deutlich größere Zersplitterung der Bildschirmfläche (als ein Anzeichen der Tendenz, möglichst viel in einer kurzen Zeit und auf einer kleinen Fläche zu vermitteln) mit Sicherheit zur Dominanz des Visuellen.

Es lassen sich zwei grundliegende Anmerkungen zum Wandel der Rolle von der Schrift (als dem semiotischen Medium) in den Nachrichtensendungen im deutschen Fernsehen formulieren. Erstens ist die Schrift zu einem wichtigeren Bestandteil der Bildschirmfläche geworden, als in den Nachrichtensendungen der 1990er Jahre. Die größere Zersplitterung der Fläche führt begünstigt die Einführung mehrerer schriftlicher Elemente, die simultan mit der audiovisuellen Übertragung angezeigt werden. Zweitens ist Schrift in den heutigen Nachrichtensendungen paradoxerweise weniger ,sprachlich“. Die simultane Anzeige und ihre zeitliche Begrenzung, die zugleich durch das technische Medium - Fernsehen und das kulturbezogene Medium - Nachrichtensendung bedingt sind, fördern die rein visuelle Betrachtung der Bildschirmfläche. Dies 
beeinträchtigt den sprachlichen Signifizierungswert der Schriftelemente bis hin zu seinem (potenziellen) völligen Verlust.

\section{Literaturverzeichnis}

Cieszkowski, Marek (2010): „O profesjonalizmach z branży ubezpieczeniowej i symulacji jako formie komunikacji”. In: Bartwicka, Halina (ed.): Język - Tekst - Kultura. Prace Komisji Językoznawczej Bydgoskiego Towarzystwa Naukowego tom XX. Bydgoszcz, Bydgoskie Towarzystwo Naukowe: 179-187.

Cieszkowski, Marek (2014): „O zasadzie równoczesności w multiprzekazie”. In: Sawicka, Grażyna/Czechowski, Wiesław (eds.): Sytuacja komunikacyjna i jej parametry. „Być nadawca - być odbiorca “. Toruń, Wydawnictwo Adam Marszałek: 40-53.

Dürscheid, Christa (2007): „Private, nicht-öffentliche und öffentliche Kommunikation im Internet". Neue Beiträge zur Germanistik 6: 22-41.

Dürscheid, Christa (2011): „Medien in den Medien - Szenen im Bild. Eine pragmatische Kommunikat-Analyse“. In: Schneider, Jan G./Stöckl, Hartmut (eds.): Medientheorien und Multimodalität. Ein TV-Werbespot - Sieben methodische Beschreibungsansätze. Köln, von Halem: $88-108$.

Fix, Ulla (2008): „Nichtsprachliches als Textfaktor: Medialität, Materialität, Lokalität““. Zeitschrift für germanistische Linguistik 36: 343-354.

Holly, Werner (2004): Fernsehen. Tübingen: Niemeyer.

Jäger, Ludwig (2001): „Sprache als Medium. Über die Sprache als audio-visuelles Dispositiv des Medialen“. In: Wenzel, Horst/Seipel, Wilfried/Wunberg, Gotthart (eds.): Audiovisualität vor und nach Gutenberg - Zur Kulturgeschichte der medialen Umbrüche. Wien, Skira: 1942.

Kapuścińska, Anna (2016): „Zum semiotischen Wert der TV-Ticker“. Zeitschrift für Literaturwissenschaft und Linguistik 46: 25-35.

Kapuścińska, Anna (2017): Grenzphänomene zwischen Text und Bild am Beispiel multimedialer Nachrichtensendungen. Berlin: Erich Schmidt.

Koch, Peter/Oesterreicher, Wulf (2011): Gesprochene Sprache in der Romania. Französisch, Italienisch, Spanisch. Berlin/New York: De Gruyter.

Posner, Roland (1985): „Nonverbale Zeichen in öffentlicher Kommunikation: Zu Geschichte und Gebrauch der Begriffe ,verbal' und ,nonverbal', ,Interaktion' und ,Kommunikation“, ,Publikum‘ und ,Öffentlichkeit‘, ,Medium‘, ,Massenmedium‘ und ,multimedial““. Zeitschrift für Semiotik 7: 235-271.

Püschel, Ulrich (1975): Semantisch-syntaktische Relationen. Untersuchungen zur Kompatibilität lexikalischer Einheiten im Deutschen. Tübingen: De Gruyter.

Saussure, Ferdinand de (1967): Grundfragen der allgemeinen Sprachwissenschaft. Berlin: de Gruyter.

Schmitz, Ulrich (2005): „Sehflächen lesen. Einführung in das Themenheft“. In: Renner, Ursula/Schmitz, Ulrich (eds.): Sehflächen lesen (=Der Deutschunterricht 57): 2-5.

Schmitz, Ulrich (2010): „Sehflächenforschung. Eine Einführung“. In: Diekmannshenke, Hajo/Klemm, Michael/Stöckl, Hartmut (eds.): Bildlinguistik. Theorien - Methoden - Fallbeispiele. Berlin, Erich Schmidt: 23-42. 
Schmitz, Ulrich (2015): Einführung in die Medienlinguistik. Darmstadt: Wissenschaftliche Buchgesellschaft.

Schneider, Jan G. (2006): „Gibt es nichtmediale Kommunikation?““. Zeitschrift für Angewandte Linguistik 44: 71-90.

Schneider, Jan Georg (2017): „Medien als Verfahren der Zeichenprozessierung. Grundsätzliche Überlegungen zum Medienbegriff und ihre Relevanz für die Gesprächsforschung“. Gesprächsforschung - Online-Zeitschrift zur verbalen Interaktion 18: 34-55.

Winkler, Hartmut (2008): Basiswissen Medien. Frankfurt am Main: Fischer. 